\title{
The Zeros of Regular Coulomb Wave Functions and of Their Derivatives
}

\author{
By Yasuhiko Ikebe
}

\begin{abstract}
A simple and efficient numerical method for computing the zeros of regular Coulomb wave functions and of their derivatives is presented. The method is based on the characterization of the zeros of the functions and of their derivatives in terms of eigenvalues of certain compact matrix operators. A similar approach has been reported for the computation of the zeros of Bessel functions and of their derivatives [9], [14].
\end{abstract}

1. Introduction. In [9], Grad and Zakrajsěk reported a matrix equation approach for the numerical computation of the zeros of Bessel functions $J_{m}(x)$ for $m \geqslant$ 0 . In [14], the method was further extended to include the zeros of $J_{m}(x)$ of any real order $m$ and of their derivatives $J_{m}^{(p)}(x)$ with certain restrictions on $m$ and $p$. In this paper, we shall show that the same approach is applicable for determining the zeros of regular Coulomb wave functions and of their first derivatives.

The regular Coulomb wave function $F_{L}(\eta, \rho)$ of order $L, L=0,1,2, \ldots$, with a real parameter $\eta,-\infty<\eta<\infty$, gives one independent solution of the Coulomb wave equation

$$
\frac{d^{2} w}{d \rho^{2}}+\left[1-\frac{2 \eta}{\rho}-\frac{L(L+1)}{\rho^{2}}\right] w=0, \quad \rho>0
$$

which is important in nuclear physics. We shall not be concerned with the irregular Coulomb wave function $G_{L}(\eta, \rho)$, which gives a second solution of (1.1). As in the case of Bessel functions [9], [14], the method is based on the characterization of the zeros in terms of eigenvalues of certain matrix operators acting in $l^{2}$, i.e., the Hilbert space of all square-summable real sequences with norm defined by

$$
\|\xi\|=\left[\sum_{n=1}^{\infty} \xi_{n}^{2}\right]^{1 / 2}, \quad \xi=\left[\xi_{1}, \xi_{2}, \ldots\right]^{T},
$$

where the symbol $T$ denotes the transpose operation. See Sections 2-3. A numerical method is derived and justified in Section 4. Actual numerical examples are presented in Section 5.

While numerical methods for computing the values of $F_{L}(\eta, \rho)$ for a given set of values, $L, \eta$, and $\rho$, are well known ([3]- [8], [10]-[13]), it appears that numerical methods for computing the zeros of $F_{L}(\eta, \rho)$ and of $d F_{L}(\eta, \rho) / d \rho$ for a given pair 
of $L$ and $\eta$ has not received sufficient attention. One could, of course, compute the zeros of $F_{L}(\eta, \rho)$ by using any one of standard root-finding methods, such as the method of bisection, the method of the false position, or the secant method, since the values of $F_{L}(\eta, \rho)$ can be readily computed using a standard method. However, such techniques require the advance knowledge of a fairly small interval containing a zero of the function, and moreover, the zeros of the function are found only one at a time. The method presented in this paper will obviate both of these difficulties.

For recent developments in the numerical methods for the evaluation of Coulomb wave functions, see [1]-[8], [10]-[13], and [17] and the references given there.

2. The Zeros of Regular Coulomb Wave Functions. For a given pair of numbers $\eta$ and $\rho$, the functions $F_{k}(\eta, \rho), k=0,1,2, \ldots$, can be characterized as a minimal solution of the linear difference equation

$$
(k+2) \sqrt{(k+1)^{2}+\eta^{2}} u_{k}-(2 k+3)\left[\eta+\frac{(k+1)(k+2)}{\rho}\right] u_{k+1}
$$

$$
+(k+1) \sqrt{(k+2)^{2}+\eta^{2}} u_{k+2}=0 .
$$

See [7, p. 63]. Dividing through by $\sqrt{2 k+3}(k+1)(k+2)$ and making the definitions

$$
\begin{gathered}
w_{k}=\sqrt{2 k+1} \cdot F_{k}(\eta, \rho), \quad k=0,1,2, \ldots, \\
e_{k}=\frac{1}{k+1} \sqrt{\frac{(k+1)^{2}+\eta^{2}}{(2 k+1)(2 k+3)}}, \quad k=0,1,2, \ldots, \\
d_{k}=\frac{1}{k(k+1)}, \quad k=1,2, \ldots,
\end{gathered}
$$

we obtain, for $k=0,1,2, \ldots$,

$$
e_{k} W_{k}-\left(\eta d_{k+1}+1 / \rho\right) W_{k+1}+e_{k+1} W_{k+2}=0 .
$$

Writing this in matrix form for $k=L, L+1, \ldots$, we have

$$
\left(T_{L, \eta}-\frac{1}{\rho} I\right) \varphi=\psi
$$

where

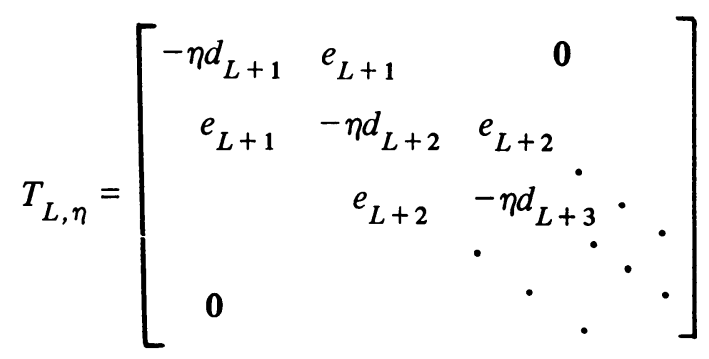

which is real, symmetric and tridiagonal, and where 


$$
\varphi=\left[W_{L+1}, W_{L+2}, \ldots\right]^{T}, \quad \psi=\left[-e_{L} w_{L}, 0,0, \ldots\right]^{T}
$$

By [7, Theorem 2.3, p. 35],

$$
\frac{2 k}{\rho} \frac{W_{k+1}}{W_{k}}=\frac{2 k}{\rho} \frac{\sqrt{2 k+3}}{\sqrt{2 k+1}} \frac{F_{k+1}}{F_{k}} \rightarrow 1 \quad(k \rightarrow \infty) .
$$

This means that the vector $\varphi$ is in the space $l^{2}$. Also,

$$
2 k \cdot e_{k} \rightarrow 1 \quad(k \rightarrow \infty) \text { and } k^{2} d_{k} \rightarrow 1 \quad(k \rightarrow \infty)
$$

This means that the sum of the squares of all elements of $T_{L, \eta}$ is finite. Hence $T_{L, \eta}$ can be regarded as a compact operator in $l^{2}$. It is also selfadjoint (real symmetric).

Theorem 2.1 (Zeros of $F_{L}(\eta, \rho)$ ). Let $L$ and $\eta$ be given. Then $\rho \neq 0$ is a zero of $F_{L}(\eta, \rho)$ if and only if $1 / \rho$ is an eigenvalue of $T_{L, \eta}$.

Proof. If $\rho \neq 0$ is a zero of $F_{L}(\eta, \rho)$, then the vector $\psi$ in (2.6) vanishes. Since $F_{L+1}(\eta, \rho) \neq 0$, the vector $\varphi$ does not vanish. Then (2.6) implies $1 / \rho$ is an eigenvalue of $T_{L, \eta}$. Conversely, let $\lambda$ be a nonzero eigenvalue of $T_{L, \eta}$. We define $\rho$ by $\lambda=1 / \rho$. Let $\left[\bar{W}_{L+1}, \bar{W}_{L+2}, \ldots\right]^{T} \in l^{2}$ be an eigenvector of $T_{L, \eta}$ corresponding to $\lambda$. Define $\bar{F}_{k}$ for $k=L, L+1, \ldots$, by

$$
\left\{\begin{array}{l}
\bar{F}_{L}=0, \\
\sqrt{2 k+1} \bar{F}_{k}=\bar{W}_{k}, \quad k=L+1, L+2, \ldots .
\end{array}\right.
$$

Then $F_{k}(\eta, \rho)$ and $\bar{F}_{k}$ satisfy the same difference equation (2.1) for $k=L, L+1, \ldots$. We have $\bar{F}_{k} \rightarrow 0$ as $k \rightarrow \infty$, since $\bar{W}_{k} \rightarrow 0$ as $k \rightarrow \infty$. This means that the $\bar{F}_{k}, k=$ $L+1, L+2, \ldots$, represent a minimal solution of (2.1). Since any pair of minimal solutions of $(2.1)$ is linearly dependent $[7$, p. 25], it follows that there exists a constant $c \neq 0$ such that

$$
F_{k}(\eta, \rho)=c \bar{F}_{k}, \quad k=L, L+1, \ldots .
$$

In particular, for $k=L, F_{L}(\eta, \rho)=c \bar{F}_{L}=0$.

COROLlaRY 2.1. The zeros of $F_{L}(\eta, \rho)$ are real.

Proof. This follows from the selfadjointness of $T_{L, \eta}$.

Remark 2.1. Consider the homogeneous equation

$$
T_{L, \eta} \varphi=0
$$

Writing $\varphi=\left[W_{L+1}, W_{L+2}, \ldots\right]^{T}$, this is equivalent to

$$
\begin{aligned}
-\eta d_{L+1} W_{L+1}+e_{L+1} W_{L+2} & =0 \\
e_{k} W_{k}-\eta d_{k+1} W_{k+1}+e_{k+1} W_{k+2} & =0, \quad k \geqslant L+1 .
\end{aligned}
$$

Assume $\eta \neq 0$. Then (see, e.g., [7, pp. 34-35]), there exists an independent solution of (2.12) such that

$$
\frac{e_{k}}{\eta d_{k}} \cdot \frac{W_{k+1}}{W_{k}} \rightarrow 1 \quad \text { as } k \rightarrow \infty
$$


i.e.,

$$
\frac{k}{\eta} \frac{W_{k+1}}{W_{k}} \rightarrow 2 \text { as } k \rightarrow \infty .
$$

This shows that, if $\eta \neq 0$, then (2.11) has a nontrivial solution in $l^{2}$. If $\eta=0$, then (2.11) reduces to

$$
\left\{\begin{array}{l}
W_{L+2}=0 \\
e_{k} W_{k}+e_{k+1} w_{k+2}=0, \quad k \geqslant L+1 .
\end{array}\right.
$$

It follows that (2.11) has only the trivial solution in $l^{2}$ if $\eta=0$, since $e_{k}>e_{k+1}$.

Remark 2.2. Let $D=\operatorname{diag}[-1,1,-1,1, \ldots]$. Then $D^{2}=I$ and

$$
D\left(-T_{L, \eta}\right) D=\left[\begin{array}{cccc}
\eta d_{L+1} & e_{L+1} & & 0 \\
e_{L+1} & \eta d_{L+2} & e_{L+2} & \\
& e_{L+2} & \eta d_{L+3} & \cdot \\
& & \cdot & \cdot \\
0 & & & \cdot
\end{array}\right]=T_{L,-\eta} .
$$

This shows that $-T_{L, \eta}$ is similar to $T_{L,-\eta}$. Consequently, the positive zeros of $F_{L}(-\eta, \rho)$ are given precisely by the absolute values of the negative zeros of $F_{L}(\eta, \rho)$. Hence, by solving an eigenvalue problem (2.6), the positive zeros of $F_{L}(\eta, \rho)$ and of $F_{L}(-\eta, \rho)$ are computed simultaneously.

Remark 2.3. If $\eta=0$, then

$$
F_{L}(\eta, \rho)=F_{L}(0, \rho)=\sqrt{\frac{\pi}{2} \cdot \rho} J_{L+(1 / 2)}(\rho) .
$$

Hence, the positive zeros of $F_{L}(0, \eta)$ are identical with those of $J_{L+(1 / 2)}(\rho)$, i.e., the Bessel function of order $L+(1 / 2)$. In particular, for $L=\eta=0$,

$$
F_{0}(0, \rho)=\sqrt{\frac{\pi}{2} \rho} J_{1 / 2}(\rho)=\sin \rho .
$$

3. The Zeros of the First Derivative of Regular Coulomb Wave Functions. We retain the notation used in Section 2. It is known that, for $L=0,1,2, \ldots$,

$$
(L+1) \frac{d F_{L}}{d \rho}=\left[\frac{(L+1)^{2}}{\rho}+\eta\right] F_{L}-\sqrt{(L+1)^{2}+\eta^{2}} F_{L+1} .
$$

Hence, $d F_{L} / d \rho=0$ if and only if

$$
\left[\frac{(L+1)^{2}}{\rho}+\eta\right] F_{L}-\sqrt{(L+1)^{2}+\eta^{2}} F_{L+1}=0 .
$$

This can be written as

$$
-\left[\frac{\eta}{(L+1)^{2}}+\frac{1}{\rho}\right] \sqrt{\frac{L+1}{2 L+1}} w_{L}+\sqrt{\frac{2 L+1}{L+1}} e_{L} w_{L+1}=0
$$


If we consider (3.3) together with the difference equation (2.5), we obtain the following matrix equation

$$
\left(\widetilde{T}_{L, \eta}-\frac{1}{\rho} I\right) \widetilde{\varphi}=0
$$

where

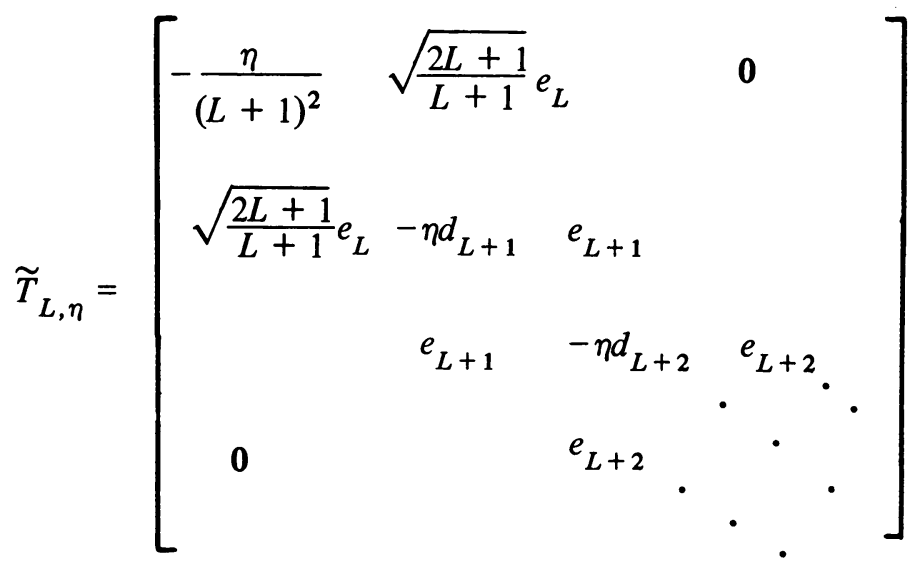

which is real, symmetric, and tridiagonal, and where

$$
\tilde{\varphi}=\left[\sqrt{\frac{L+1}{2 L+1}} w_{L}, w_{L+1}, W_{L+2}, \ldots\right]^{T} .
$$

Just like $T_{L, \eta}$ in Section 2, $\widetilde{T}_{L, \eta}$ is a compact operator in $l^{2}$.

Theorem 3.1 (ZERos of $\left.d F_{L} / d \rho\right)$. Let $L$ and $\eta$ be given. Then $\rho \neq 0$ is a zero of $d F_{L}(\eta, \rho) / d \rho$ if and only if $1 / \rho$ is an eigenvalue of $\widetilde{T}_{L, \eta}$.

The proof is similar to that of Theorem 2.1 and is omitted.

COROLlary 3.1. The zeros of $d F_{L} / d \rho$ are real.

Remark 3.1. As for $T_{L, \eta}, 0$ is an eigenvalue of $\widetilde{T}_{L, \eta}$ when $\eta \neq 0$, and is not when $\eta=0$.

Remark 3.2. The positive zeros of $d F_{L}(-\eta, \rho) / d \rho$ are given precisely by the absolute values of the negative zeros of $d F_{L}(\eta, \rho) / d \rho$. See Remark 2.2.

4. The Numerical Procedure. A numerical method for computing the zeros of $F_{L}(\eta, \rho)$ and of $d F_{L}(\eta, \rho) / d \rho$ for a given pair of numbers $L$ and $\eta$ is now presented.

(I) Zeros of $F_{L}(\eta, \rho)$. Let $n$ be a positive integer. Let $T_{L, \eta}^{(n)}$ denote the principal $n \times n$ submatrix of $T_{L, \eta}$. We shall refer to $T_{L, \eta}^{(n)}$ as the truncated matrix of order $n$. It is known that the nonvanishing zeros of $F_{L}(\eta, \rho)$ are simple. Hence, by Theorem 2.1, all nonzero eigenvalues of $T_{L, \eta}$ are simple. By the Sturm-sequence theorem [16, pp. 299-302], the eigenvalues of $T_{L, \eta}^{(n)}$ are also simple. Let $\lambda_{-1}<\lambda_{-2}<\ldots<$ $0<\ldots<\lambda_{2}<\lambda_{1}$ be a complete enumeration of the nonzero eigenvalues of $T_{L, \eta}$. Let $\lambda_{-1}^{(n)}<\lambda_{-2}^{(n)}<\ldots<0<\ldots<\lambda_{2}^{(n)}<\lambda_{1}^{(n)}$ be a complete enumeration of the eigenvalues of $T_{L, \eta}^{(n)}$. The zeros of $F_{L}(\eta, \rho)$ are approximated by $1 / \lambda_{k}^{(n)}$, where $\lambda_{k}^{(n)} \neq$ 0. By [13, pp. 279-281], we have the following estimate for any $k$ :

$$
\left|\lambda_{k}^{(n)}-\lambda_{k}\right| \leqslant\left\|T_{L, \eta}^{(n)}-T_{L, \eta}\right\| \quad \text { (operator norm). }
$$


TABLE 1

The least 5 positive zeros of $F_{L}(\eta, \rho)$ correct to 10 figures

\begin{tabular}{|c|c|c|c|c|c|}
\hline \multicolumn{6}{|c|}{$\mathrm{L}=0$} \\
\hline$\eta=0$ & & $\eta=1$ & & $\eta=2$ & \\
\hline 3.141592654 & $(00)$ & 5.814115616 & $(00)$ & 8.395670124 & $(00)$ \\
\hline 6.283185307 & $(00)$ & 9.474533918 & $(00)$ & 1.240524258 & $(01)$ \\
\hline 9.424777961 & $(00)$ & 1.294165270 & (01) & 1.611044740 & $(01)$ \\
\hline 1.256637061 & (01) & 1.632324836 & (01) & 1.967631893 & $(01)$ \\
\hline 1.570796327 & (01) & 1.965575668 & $(01)$ & 2.316032851 & $(01)$ \\
\hline$\eta=4$ & & $\eta=8$ & & $\eta=16$ & \\
\hline 1.321926777 & (01) & 2.232333211 & (01) & 3.976616365 & (01) \\
\hline 1.774290736 & (01) & 2.757188361 & (01) & 4.600224064 & (01) \\
\hline 2.181535538 & (01) & 3.218280961 & $(01)$ & 5.136931880 & (01) \\
\hline 2.567409274 & (01) & 3.648271962 & (01) & 5.630269889 & (01) \\
\hline 2.940417556 & (01) & 4.059123272 & (01) & 6.096436277 & (01) \\
\hline \multicolumn{6}{|c|}{$L=1$} \\
\hline$\eta=0$ & & $\eta=1$ & & $\eta=2$ & \\
\hline 4.493409458 & $(00)$ & 6.566570904 & $(00)$ & 8.851065605 & $(00)$ \\
\hline 7.7252 .51837 & $(00)$ & 1.023885720 & (01) & 1.286321977 & $(01)$ \\
\hline 1.090412166 & (01) & 1.371133324 & (01) & 1.656969608 & (01) \\
\hline 1.406619391 & (01) & 1.709605052 & (01) & 2.013634835 & $(01)$ \\
\hline 1.722075527 & (01) & 2.043062027 & $(01)$ & 2.362089262 & (01) \\
\hline$\eta=4$ & & $\eta=8$ & & $\eta=16$ & \\
\hline 1.346275189 & (01) & 2.244745611 & (01) & 3.982854985 & (01) \\
\hline 1.798676787 & (01) & 2.769605220 & $(01)$ & 4.606463125 & $(01)$ \\
\hline 2.205942254 & (01) & 3.230700483 & $(01)$ & 5.143171234 & (01) \\
\hline 2.591829573 & (01) & 3.660693361 & (01) & 5.636509465 & (01) \\
\hline 2.964847623 & (01) & 4.071546094 & $(01)$ & 6.102676029 & (01) \\
\hline
\end{tabular}

The bound on the right-hand side of the last inequality converges to 0 as $n \rightarrow \infty$, but the bound is usually too pessimistic. In practice, it is enough to observe the numerical convergence $\lambda_{k}^{(n)} \rightarrow \lambda_{k}$.

(II) A similar procedure applies in the numerical computation of the zeros of $d F_{L}(\eta, \rho) / d \rho$. Thus, if $\widetilde{T}_{L, \eta}^{(n)}$ denotes the principal $n \times n$ submatrix of $\widetilde{T}_{L, \eta}$, then the zeros of $F_{L}(\eta, \rho)$ are approximated by $1 \tilde{\lambda}_{k}^{(n)}$, where $\tilde{\lambda}_{k}^{(n)}$ represents an arbitrary nonvanishing eigenvalue of $\widetilde{T}_{L, \eta}^{(n)}$. 
TABLE 2

The least 5 positive zeros of $d F_{L}(\eta, \rho) / d \rho$ correct to 10 figures

\begin{tabular}{|c|c|c|c|c|c|}
\hline \multicolumn{6}{|c|}{$L=0$} \\
\hline$\eta=0$ & & $\eta=1$ & & $\eta=2$ & \\
\hline 1.570796327 & $(00)$ & 3.657410638 & $(00)$ & 5.895085350 & $(00)$ \\
\hline 4.712388980 & $(00)$ & 7.667677779 & $(00)$ & 1.043657415 & (01) \\
\hline 7.853981634 & $(00)$ & 1.121679693 & $(01)$ & 1.427184702 & (01) \\
\hline 1.099557429 & (01) & 1.463706308 & $(01)$ & 1.790100351 & (01) \\
\hline 1.413716694 & $(01)$ & 1.799237821 & $(01)$ & $2.1423,16801$ & (01) \\
\hline$\eta=4$ & & $\eta=8$ & & $\eta=16$ & \\
\hline 1.025063372 & $(01)$ & 1.873288298 & $(01)$ & 3.536434308 & (01) \\
\hline 1.553379997 & $(01)$ & 2.502084729 & $(01)$ & 4.298288977 & (01) \\
\hline 1.980033171 & $(01)$ & 2.990784803 & $(01)$ & 4.872792458 & (01) \\
\hline 2.375658102 & $(01)$ & 3.435024172 & $(01)$ & 5.386063072 & (01) \\
\hline 2.754682974 & $(01)$ & 3.854853936 & $(01)$ & 5.865008314 & (01) \\
\hline & & $L=1$ & & & \\
\hline$\eta=0$ & & $\eta=1$ & & $\eta=2$ & \\
\hline 2.743707270 & $(00)$ & 4.38750385 .1 & $(00)$ & 6.346301319 & $(00)$ \\
\hline 6.116764264 & $(00)$ & 8.426947553 & $(00)$ & 1.089342998 & (01) \\
\hline 9.316615629 & $(00)$ & 1.198404802 & $(01)$ & 1.473051307 & (01) \\
\hline 1.248593737 & $(01)$ & 1.540841197 & $(01)$ & 1.836066699 & (01) \\
\hline 1.564386611 & $(01)$ & 1.876626782 & $(01)$ & 2.188347826 & (01) \\
\hline$\eta=4$ & & $\eta=8$ & & $\eta=16$ & \\
\hline 1.049361433 & (01) & 1.885695887 & $(01)$ & 3.542672491 & (01) \\
\hline 1.577738308 & $(01)$ & 2.514499607 & $(01)$ & 4.304527833 & (01) \\
\hline 2.004430293 & $(01)$ & 3.003203073 & $(01)$ & 4.879021673 & $(01)$ \\
\hline 2.400071979 & (01) & 3.447444675 & (01) & 5.392302541 & (01) \\
\hline 2.779108373 & $(01)$ & 3.867276073 & $(01)$ & 5.871247981 & (01) \\
\hline
\end{tabular}

5. Examples. Some of our computational results follow.

Tables 1 and 2 show the first five positive zeros of $F_{L}(\eta, \rho)$ and of $d F_{L}(\eta, \rho) / d \rho$ correct to 10 decimal figures, for $L=0$ and 1 and $\eta=0,1,2,4,8$, and 16 . In practical applications of the regular Coulomb wave functions, $\eta$ is usually positive.

Table 3 shows the first positive zeros of $F_{L}(\eta, \rho)$ and of $d F_{L}(\eta, \rho) / d \rho$ correct to 10 decimal figures for $L=1$ and $\eta=-1$, as an example of the case of negative $\eta$. This requires no new computations as stated in Remark 2.2. 
TABLE 3

The least 5 positive zeros of $F_{1}(-1, \rho)$ and of $d F_{1}(-1, \rho) / d \rho$, correct to 10 figures

\begin{tabular}{|ll|ll|}
\hline \multicolumn{2}{|c|}{$F_{1}(-1, \rho)$} & \multicolumn{3}{|c|}{$\mathrm{dF}_{1}(-1, \rho) / d \rho$} \\
\hline 2.966144623 & $(00)$ & 1.680442277 & $(00)$ \\
5.661920828 & $(00)$ & 4.302520276 & $(00)$ \\
8.478875692 & $(00)$ & 7.063549724 & $(00)$ \\
1.136894161 & $(01)$ & 9.919630533 & $(00)$ \\
1.430633125 & $(01)$ & 1.283475614 & $(01)$ \\
\hline
\end{tabular}

TABLE 4

The number of computed positive zeros of $F_{L}(\eta, \rho)$ correct to 10 or more figures

\begin{tabular}{|c|c|c|c|c|c|c|}
\hline \multirow[b]{2}{*}{$\eta$} & \multicolumn{3}{|c|}{$L=0$} & \multicolumn{3}{|c|}{$L=1$} \\
\hline & $n=32$ & $n=64$ & $\mathrm{n}=128$ & $n=32$ & $n=64$ & $n=128$ \\
\hline $\begin{array}{r}16 \\
8 \\
4 \\
2 \\
1\end{array}$ & $\begin{array}{l}0 \\
1 \\
3 \\
4 \\
5\end{array}$ & $\begin{array}{r}5 \\
8 \\
11 \\
13 \\
14\end{array}$ & $\begin{array}{l}21 \\
26 \\
29 \\
31 \\
33\end{array}$ & $\begin{array}{l}0 \\
1 \\
3 \\
4 \\
5\end{array}$ & $\begin{array}{r}5 \\
9 \\
11 \\
13 \\
14\end{array}$ & $\begin{array}{l}21 \\
26 \\
29 \\
31 \\
32\end{array}$ \\
\hline 0 & 6 & 15 & 34 & 6 & 15 & 34 \\
\hline $\begin{array}{r}-1 \\
-2 \\
-4 \\
-8 \\
-16\end{array}$ & $\begin{array}{r}7 \\
8 \\
9 \\
11 \\
13\end{array}$ & $\begin{array}{l}16 \\
17 \\
19 \\
22 \\
25\end{array}$ & $\begin{array}{l}36 \\
37 \\
39 \\
43 \\
47\end{array}$ & $\begin{array}{r}7 \\
7 \\
8 \\
10 \\
12\end{array}$ & $\begin{array}{l}15 \\
17 \\
19 \\
21 \\
24\end{array}$ & $\begin{array}{l}35 \\
37 \\
39 \\
42 \\
47\end{array}$ \\
\hline
\end{tabular}

TABLE 5

The number of computed positive zeros of $d F_{L}(\eta, \rho) / d \rho$ correct to 10 or more figures

\begin{tabular}{|c|c|c|c|c|c|c|}
\hline$\eta$ & \multicolumn{3}{|c|}{$\mathrm{L}=0$} & \multicolumn{3}{c|}{$\mathrm{L}=1$} \\
\cline { 2 - 7 } & $\mathrm{n}=32$ & $\mathrm{n}=64$ & $\mathrm{n}=128$ & $\mathrm{n}=32$ & $\mathrm{n}=64$ & $\mathrm{n}=128$ \\
\hline 8 & 0 & 5 & 21 & 0 & 5 & 21 \\
4 & 2 & 9 & 26 & 2 & 9 & 26 \\
2 & 3 & 11 & 30 & 3 & 11 & 30 \\
1 & 4 & 12 & 32 & 5 & 12 & 32 \\
\hline 0 & 5 & 14 & 33 & 5 & 14 & 33 \\
\hline-1 & 7 & 15 & 35 & 6 & 15 & 34 \\
-2 & 8 & 18 & 36 & 7 & 16 & 36 \\
-4 & 9 & 19 & 37 & 8 & 17 & 37 \\
-8 & 11 & 22 & 40 & 9 & 19 & 39 \\
-16 & 13 & 25 & 48 & 11 & 21 & 42 \\
\hline
\end{tabular}


Tables 4 and 5 show how the number of computed positive zeros of $F_{L}(\eta, \rho)$ and of $d F_{L}(\eta, \rho) / d \rho$, correct to 10 or more figures, depends on $L, \eta$, and $n$, where $n$ denotes the order of the truncated matrices $T_{L, \eta}^{(n)}$ or $\widetilde{T}_{L, \eta}^{(n)}$ (see Section 4). For instance, Table 4 indicates that for $L=1$ and $\eta=1$ the use of the truncated matrix of order $n=64$ produces the first 14 positive zeros of $F_{L}(\eta, \rho)$, correct to 10 or more significant figures. The number was determined by comparing the computed zeros for successive values of $n$.

The computations were done on the CDC $6600 / 6400$ system at The University of Texas at Austin, using single-precision (net 48-bit) floating-point arithmetic (FORTRAN REAL arithmetic). For the computation of the eigenvalues of real symmetric tridiagonal matrices, the FORTRAN subroutine IMTQLI in EISPACK [16] was used. The computing time (the CDC 6600 central processor time) depends on $n$. About 0.86 seconds were needed for $n=64,2.0$ seconds for $n=128$, and 6.6 seconds for $n=256$.

Note. In the above tables, the numbers appearing in parentheses represent the exponent relative to base 10. For instance, 1.321926777 (01), i.e., the first positive zero of $F_{L}(\eta, \rho)$ for $L=0$ and $\eta=4$ (Table 1 ), means $1.321926777 \times 10^{1}$.

Acknowledgment. The author wishes to express his appreciation to $\mathrm{Mr}$. V. Benokraitis for reading the manuscript and suggesting many improvements, and to the referee for pointing out further improvements.

Center for Numerical Analysis The University of Texas at Austin Austin, Texas 78712

1. M. ABRAMOWITZ \& I. A. STEGUN (Editors), Handbook of Mathematical Functions, With Formulas, Graphs and Mathematical Tables, reprint, Dover, New York, 1966. MR 34 \#8606.

2. W. J. CODY \& K. HILLSTROM, "Chebyshev approximations for the Coulomb phase shift," Math. Comp., v. 24, 1970, pp. 671-677. MR 42 \#661.

3. W. J. CODY \& KATHLEEN A. PACIOREK, "Remark on algorithm 292-regular Coulomb wave functions," Comm. ACM, v. 13, 1970, p. 573.

4. A. R. CURTIS, Coulomb Wave Functions, Royal Soc. Math. Tables, vol. 11, Cambridge Univ. Press, New York, 1964. MR 29 \#4915.

5. W. GAUTSCHI, "Algorithm 292-regular Coulomb wave functions," Comm. ACM, v. 9, 1966, pp. 793-795.

6. W. GAUTSCHI, "Remark on algorithm 292-regular Coulomb wave functions," Comm. $A C M$, v. 12, 1969, p. 280.

7. W. GAUTSCHI, "Computational aspects of three-term recurrence relations," SIAM Rev., v. 9, 1967, pp. 24-82. MR 35 \#3927.

8. W. GAUTSCHI, "An application of minimal solutions of three-term recurrences to Coulomb wave functions," Aequationes Math., v. 2, 1969, pp. 171-176. MR 39 \#716.

9. J. GRAD \& E. ZAKRAJSĚK, "Method for evaluation of zeros of Bessel functions," J. Inst. Math. Appl., v. 11, 1973, pp. 57-72.

10. J. H. GUNN, "Algorithm 300-Coulomb wave functions," Comm. $A C M$, v. 10, 1967, pp. 244-245.

11. K. S. KÖLBIG, "Certification of algorithm 300-Coulomb wave functions," Comm. $A C M$, v. 12, 1969, pp. 279-280.

12. K. S. KÖLBIG, "Certification algorithm 292-regular Coulomb wave functions," Comm. $A C M$, v. 12, 1969, pp. 278-279.

13. K. S. KÖLBIG, "Remarks on the computation of Coulomb wave functions," Computer Physics Comm., v. 4, 1972, pp. 214-220. 
14. Y. IKEBE, The Zeros of Bessel Functions and of Their Derivatives, Technical Report CNA-81, Center for Numerical Analysis, The University of Texas, Austin, Texas, February 1974. 15. M. A. KRASNOSEL'SKIĬ, G. M. VAĬNIKKO, P. P. ZABREĬKO, Ja. B. RUTICKIĬ \& V. Ja. STECENKO, Approximate Solution of Operator Equations, "Nauka", Moscow, 1969; English transl., Wolters-Noordhoff, Groningen, 1972. MR 41 \#4271.

16. B. T. SMITH, J. M. BOYLE, B. S. GARBOW, Y. IKEBE, C. KLEMA \& C. B. MOLER, Matrix Eigensystem Routines-EISPACK Guide, Springer-Verlag, New York, 1974.

17. A. J. STRECOK \& J. A. GREGORY, "High precision evaluation of the irregular Coulomb wave functions," Math. Comp., v. 26, 1972, pp. 955-961. MR 47 \#2791.

18. J. H. WILKINSON, The Algebraic Eigenvalue Problem, Clarendon Press, Oxford, 1965. MR 32 \#1894. 